\title{
Structure-activity Relationships of Amyloid Beta-aggregation Inhibitors Based on Curcumin: Influence of Linker Length and Flexibility
}

\author{
Ashley A. Reinke and Jason E. Gestwicki* \\ Department of Pathology and the Life Sciences Institute, University \\ of Michigan, Ann Arbor, Michigan 48109-2216, USA \\ *Corresponding author: Jason E. Gestwicki, gestwick@umich.edu
}

\begin{abstract}
Self-assembly of amyloid beta into fibrillar plaques is characteristic of Alzheimer's disease and oligomers of this peptide are believed to be involved in neurodegeneration. Natural organic dyes, such as congo red and curcumin, bind tightly to amyloid beta and, at higher concentrations, block its selfassembly. The ability of these molecules to prevent amyloid accumulation has generated interest in understanding which of their structural features contribute to inhibitory potency. In general, amyloid beta ligands tend to be flat, planar molecules with substituted aromatic end groups; however, a comprehensive structure-activity study has not been reported. To better understand these ligands, we surveyed the effect of three prominent features on inhibition of amyloid aggregation: the presence of two aromatic end groups, the substitution pattern of these aromatics, and the length and flexibility of the linker region. We found that modification of any one of the modules has profound effects on activity. Further, we report that the optimal length of the linker lies within a surprisingly narrow regime (6-19 $\AA$ ). These results offer insight into the key chemical features required for inhibiting amyloid beta aggregation. In turn, these findings help define the nature of the docking site for small molecules on the amyloid beta surface.
\end{abstract}

Key words: Alzheimer's disease, neurodegeneration, protein misfolding, thioflavin T

Received 10 July 2007, revised 20 July 2007 and accepted for publication 21 July 2007

The aggregation of amyloid beta $(A \beta)$ peptide has been implicated in the pathology of patients with Alzheimer's disease (1-3). A $\beta$ peptides are typically 39-42 residues long and, in vitro, they will spontaneously self-assemble into fibrillar and oligomeric structures that are reminiscent of material isolated from the diseased brain (4-9). Recent evidence points to oligomers as the key neurotoxin
(10-12) and, thus, significant efforts have been placed toward identifying small, drug-like molecules that block early stages of amyloid self-assembly (13-16). X-ray diffraction, electron microscopy, and solid-state NMR studies suggest that $A \beta$ multimers are stabilized by hydrophobic and hydrogen-bonding interactions within the $\beta$-sheets that form their core (17-22). Although this general model is well supported, a detailed molecular understanding of the key toxin remains elusive. Thus, the structure-guided design of small molecules that disrupt the forces favoring $A \beta$ self-assembly has been challenging.

One approach to identifying potent inhibitors is to pattern them after naturally occurring compounds. Organic dyes, such as congo red (CR), chrysamine $\mathrm{G}(\mathrm{CG})$, and curcumin, bind with high affinity to $A \beta(23-27)$. Additionally, curcumin, an abundant component of the plant turmeric that is used in the curry spice of the same name, has been shown to inhibit $A \beta(1-40)$ fibril formation and lower its toxicity $(26,28)$. Interestingly, curcumin, CR, and CG all share a similar chemical scaffold; they contain two substituted aromatic groups separated by a rigid, planar backbone (Figure 1A). Consistent with the importance of this core, several groups have reported that other roughly curcumin-like ligands are also inhibitors of $A \beta$ aggregation (29-38). Despite these advances, the structure-activity relationships that define potent ligands have not been systematically explored. We hypothesize that a better understanding of the modules necessary for activity would facilitate creation of new inhibitors. In turn, these insights might reveal the key elements of the amyloid surface that are required for its aggregation and toxicity.

To identify the chemical features most important for inhibition, we have constructed a library of small molecules. To simplify the study, we have focused on molecules that resemble curcumin and CR; thus, benzothiazoles and related molecules, which are believed to bind a site on the $A \beta$ surface distinct from where $C R$ interacts, were not included (39). This collection consists of both known curcumin-inspired ligands and novel synthetic compounds that we generated to test the contribution of specific substructural elements. Collectively, this library addresses three components predicted to impact activity (Figure 1B). First, we hypothesized that the chemical scaffold of curcumin, containing two aromatic end groups, is optimal for inhibition and the $R_{1}$ component addresses whether compounds that lack the second aromatic group retain activity. Secondly, we predicted that substitutions on the phenyl groups are essential to activity; therefore, the $R_{2}$ study addresses the effect of altering the hydrogen-bonding properties of these substitutions. 
(A)<smiles>COc1cc(/C=C/C(=O)CC(=O)/C=C/c2ccc(O)c(O)c2)ccc1O</smiles><smiles>Nc1c(N=Nc2ccc(-c3ccc(N=Nc4cc([N+](=O)[O-])c5ccccc5c4N)cc3)cc2)cc([N+](=O)[O-])c2ccccc12</smiles>

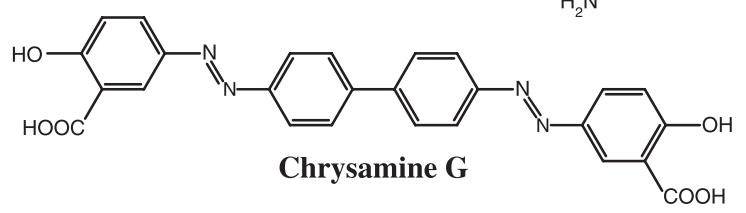

(B) Substitutions Linker region $2^{\text {nd }}$ aromatic group

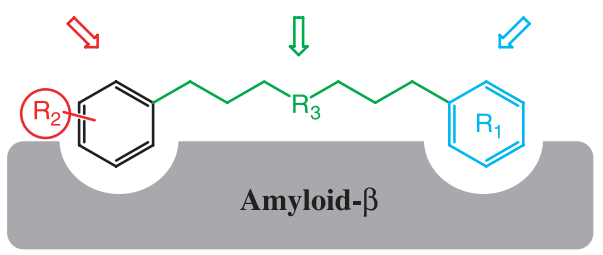

Figure 1: The structural components of natural amyloid beta $(A B)$ ligands. (A) Chemical structures of curcumin and related compounds. (B) The structural elements common to curcumin-like amyloid ligands. These features are shown in relation to a schematic $A \beta$ surface to illustrate how these different components may contribute to molecular recognition.

Finally, the $R_{3}$ study examines the effects of linker length and flexibility. Overall, we show that modest changes in any of the $R_{1}$. $R_{2}$, or $R_{3}$ components have profound effects on activity. This finding emphasizes the strict requirements for inhibition and these constraints lend themselves to a general model of inhibition by curcumin and related ligands.

\section{Methods and Materials}

\section{General}

All chemical reagents were purchased from Sigma-Aldrich (St. Louis, MD, USA) and were used without purification. Library members whose synthesis is not described below were purchased from Sigma-Aldrich. Solvents were purchased from Thermofisher Corp. (Waltham, MA, USA). All mass spectrometry analysis was carried out on a Waters LCT Electrospray-TOF instrument in positive ion mode (Waters, Milford, MA, USA). NMR spectra were recorded on a Bruker Avance DRX500 NMR Spectrometer running TOPSPIN 1.3 Software (Bruker, Billerica, MA, USA). Yields and purities for each compound are summarized in Table 1.

\section{Synthesis}

Compounds $\mathbf{6}$ and $\mathbf{7}$ were prepared via an aldol reaction based on previous findings (40). Briefly, acetophenone $(5 \mathrm{mmol})$ was added to
$10 \mathrm{~mL}$ 9:1 ethanol:water and stirred at $0{ }^{\circ} \mathrm{C}$ for $10 \mathrm{~min}$. About $25 \mathrm{mmol} \mathrm{NaOH}$ was added and the reaction was allowed to stir for another $10 \mathrm{~min}$ at $0{ }^{\circ} \mathrm{C} .5 \mathrm{mmol}$ of the appropriate aldehyde was added and the reaction proceeded for $17 \mathrm{~h}$ at room temperature. Each reaction was extracted twice with dichloromethane (DCM) and brine addition. Products were characterized by mass spectrometry and NMR. The 4-hydroxy-3-methoxy benzaldehyde (vanillin)-based chalcone (6) afforded a brown oil (yield $14 \%$ ) at a $>95 \%$ purity [exact mass calculated for $\mathrm{C}_{16} \mathrm{H}_{14} \mathrm{O}_{3}$ : 254.3, observed $(\mathrm{M}+\mathrm{H})$ : 255.1, 100\%]. The 3-hydroxybenzaldehyde-based chalcone (7) formed a light brown solid (yield 17\%) at a $>95 \%$ purity [exact mass calculated for $\mathrm{C}_{15} \mathrm{H}_{12} \mathrm{O}_{2}: 224.3$, observed $\left.(\mathrm{M}+\mathrm{H}): 225.1,100 \%\right]$

Compounds 11-14 were prepared via amide coupling of ferulic acid (5) and various diamines. Synthesis was patterned after the approach of Spasova et al. (41). Ferulic acid [(E)-3-(4-hydroxy-3methoxyphenyl)prop-2-enoic acid; $3 \mathrm{mmol}]$ was dissolved in 6-10 mL 1:1 dimethylformamide:DCM, followed by the addition of triethylamine $(0.75 \mathrm{mmol})$. The reaction was allowed to stir for $10 \mathrm{~min}$ at $0{ }^{\circ} \mathrm{C}$. $\mathrm{N}$-Hydroxybenzotriazole $(3 \mathrm{mmol})$ and $\mathrm{N}_{1} \mathrm{~N}^{\prime}$-diisopropylcarbodiimide $(8 \mathrm{mmol})$ were added directly to the mixture. About $1.5 \mathrm{mmol}$ of $\mathrm{N}$-methylmorpholine dissolved in $\mathrm{DCM}(2 \mathrm{~mL})$ was added and stirred at $0{ }^{\circ} \mathrm{C}$ for another $7 \mathrm{~min}$. $0.75 \mathrm{mmol}$ of either ethylenediamine, diaminobutane, diaminooctane, or $m$-xylylenediamine was dissolved in 2-3 mL DCM and slowly added to the reaction mixture. The reactions proceeded at room temperature for $23 \mathrm{~h}$ (ethylenediamine, diaminobutane, and diaminooctane reactions) or $46 \mathrm{~h}$ (m-xylylenediamine reaction), after which 1 eq. of $5 \% \mathrm{NaH}$ $\mathrm{CO}_{3}$ was added. Upon agitation, an orange to brown solid precipitated. In each case, the solid was obtained by filtration and washed with cold water. Each solid was then dissolved in a 1:1 methanol/DCM mixture, and precipitated a second time with brine. Filtration of this solid yielded the final products. Compounds 11-14 were characterized by mass spectrometry and ${ }^{1} \mathrm{H}-\mathrm{NMR}$.

Compound 11 (2E,2'E-N,N' -(ethane-1,2-diy))bis(3-(4-hydroxy-3-methoxyphenyl)prop-2-enamide) (yield 16\%): Exact mass calculated for $\mathrm{C}_{22} \mathrm{H}_{24} \mathrm{~N}_{2} \mathrm{O}_{6}:$ : 412.4, observed $\left(\mathrm{M}+\mathrm{Na}^{+}\right): 435.1$ (20\%). ${ }^{1} \mathrm{H}-\mathrm{NMR}$ (500 MHz; DMSO), $\delta 3.28(s, 4 H), \delta 3.80(\mathrm{~s}, 6 \mathrm{H}), \delta 6.43(\mathrm{~d}, 2 \mathrm{H}), \delta 6.78$ $(\mathrm{d}, 2 \mathrm{H}), \delta 6.98(\mathrm{~d}, 2 \mathrm{H}), \delta 7.10(\mathrm{~s}, 2 \mathrm{H}), \delta 7.33(\mathrm{~d}, 2 \mathrm{H}), \delta 8.1$ (s, 2H).

Compound 12 (2E,2'E-N,N'N-(butane-1,4-diy))bis(3-(4-hydroxy-3-methoxyphenyl)prop-2-enamide) (yield 15\%): Exact mass calculated for $\mathrm{C}_{24} \mathrm{H}_{28} \mathrm{~N}_{2} \mathrm{O}_{6}: 440.2$, observed $\left(\mathrm{M}+\mathrm{Na}^{+}\right): 463.2(100 \%)$. ${ }^{1} \mathrm{H}-\mathrm{NMR}$ (500 MHz; DMSO), $\delta 1.47$ (s, 4H), $\delta 3.17(\mathrm{~s}, 4 \mathrm{H}), \delta 3.78$ (s, 6H), $\delta$ $6.39(\mathrm{~d}, 2 \mathrm{H}), \delta 6.73(\mathrm{~d}, 2 \mathrm{H}, \delta 6.93(\mathrm{~d}, 2 \mathrm{H}), \delta 7.06(\mathrm{~s}, 2 \mathrm{H}), \delta 7.29$ (d, $2 \mathrm{H}, \delta 7.98(\mathrm{~s}, 2 \mathrm{H})$.

Compound 13 (2E,2'E-N,N'N-(octane-1,8-diy))bis(3-(4-hydroxy-3-methoxyphenyl)prop-2-enamide) (yield 8\%): Exact mass calculated for $\mathrm{C}_{28} \mathrm{H}_{36} \mathrm{~N}_{2} \mathrm{O}_{6}$ : 496.3, observed $\left(\mathrm{M}+\mathrm{Na}^{+}\right): 519.2$ (100\%). ${ }^{1} \mathrm{H}-\mathrm{NMR}$ (500 MHz; DMSO), $\delta 1.29$ (s, 8H, $\delta 1.44(\mathrm{~s}, 4 \mathrm{H}, \delta 3.14$ (s, 4H), $\delta 3.78(\mathrm{~s}, 6 \mathrm{H}), \delta 6.38(\mathrm{~d}, 2 \mathrm{H}), \delta 6.74(\mathrm{~d}, 2 \mathrm{H}), \delta 6.94(\mathrm{~d}, 2 \mathrm{H}), \delta 7.06$ $(\mathrm{s}, 2 \mathrm{H}), \delta 7.28(\mathrm{~d}, 2 \mathrm{H}), \delta 7.91$ (s, 2H).

Compound 14 (2E,2'E)-N, $N^{\prime}$-(1,3-phenylenebis(methylene))bis(3-(4hydroxy-3-methoxyphenyl)prop-2-enamide) (yield 19\%): Exact mass calculated for $\mathrm{C}_{28} \mathrm{H}_{28} \mathrm{~N}_{2} \mathrm{O}_{6}$ : 488.5, observed $\left(\mathrm{M}+\mathrm{Na}^{+}\right): 511.1$ 
Reinke and Gestwicki

Table 1: Structure-activity relationships library: compounds tested for inhibition of amyloid beta aggregation

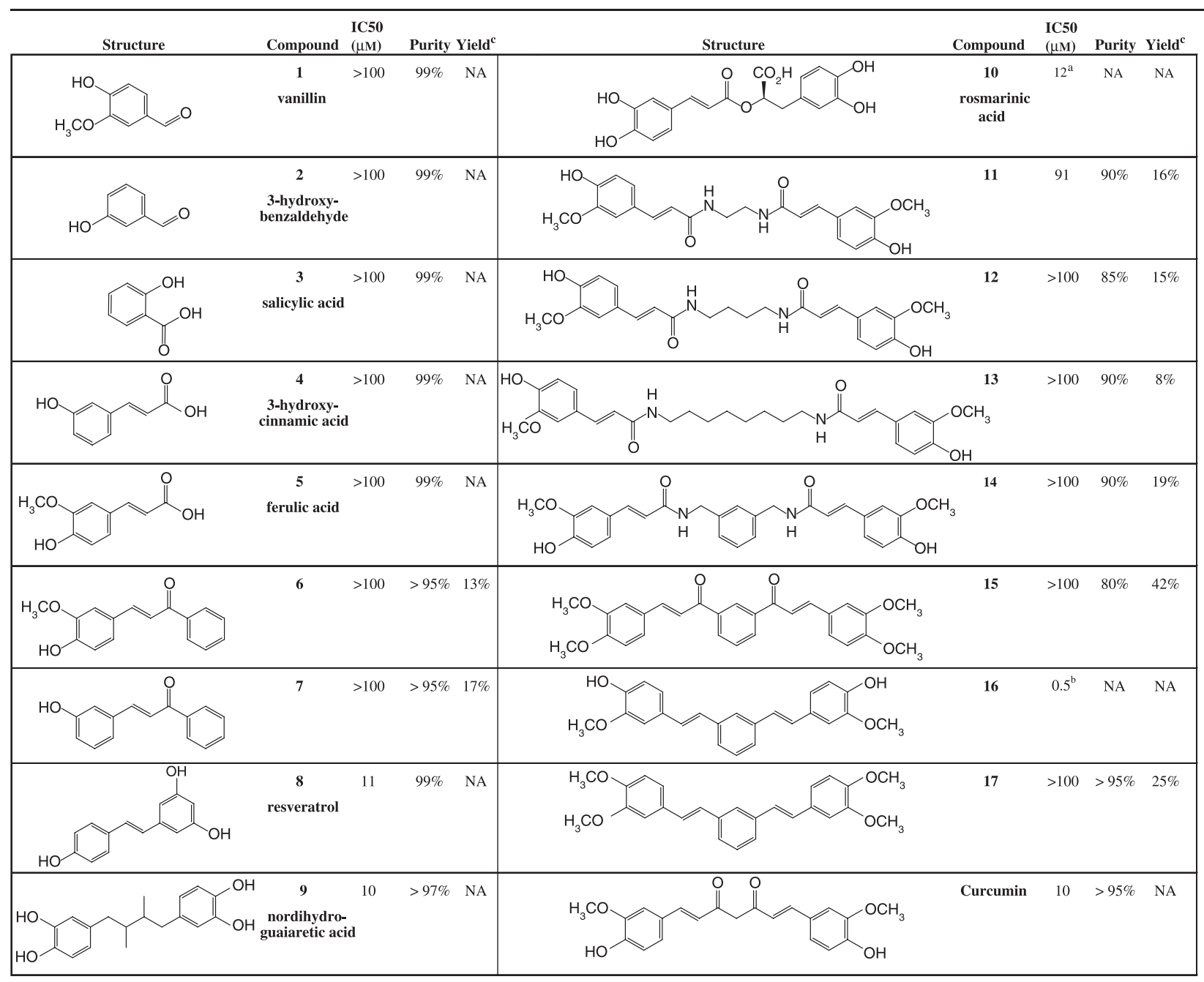

${ }^{\mathrm{a}} \mathrm{C}_{50}$ value reported by Masuda et al. (35).

${ }^{\mathrm{b}} \mathrm{C}_{50}$ value reported by Lee et al. (32).

${ }^{c}$ Compound labeled NA were purchased from Sigma and used without further purification.

(100\%). ${ }^{1} \mathrm{H}-\mathrm{NMR}$ (500 MHz; DMSO), $\delta 3.78(\mathrm{~s}, 6 \mathrm{H}), \delta 4.37$ (s, 4H), $\delta 6.47(\mathrm{~d}, 2 \mathrm{H}), \delta 6.75(\mathrm{~d}, 2 \mathrm{H}), \delta 6.95(\mathrm{~d}, 2 \mathrm{H}), \delta 7.08(\mathrm{~s}, 2 \mathrm{H}), \delta 7.17$ $(\mathrm{d}, 2 H), \delta 7.22(\mathrm{~s}, 1 H), \delta 7.29(\mathrm{t}, 1 H), \delta 7.35(\mathrm{~d}, 2 H), \delta 8.47(\mathrm{t}, 2 H)$.

Compound 15 (2E,2'E)-1,1'-(1,3-phenylene)bis(3-(3,4-dimethoxyphenyl)prop-2-en-1-one) was formed via an aldol reaction. 1,3-Diacetylbenzene $(1.4 \mathrm{mmol})$ was added to $25 \mathrm{~mL}$ ethanol and stirred for $10 \mathrm{~min}$ at $0{ }^{\circ} \mathrm{C} .14 .4 \mathrm{mmol} \mathrm{NaOH}$ was added and the reaction stirred for another $10 \mathrm{~min}$ at $0^{\circ} \mathrm{C}$. $8.3 \mathrm{mmol}$ of 3,4-dimethoxybenzaldehyde (veratraldehyde) was added and the reaction proceeded at room temperature for $13 \mathrm{~h}$. About $5 \mathrm{~mL}$ water was added to promote precipitation of a yellow solid. The mixture was vacuum-filtered and washed with cold water to afford a bright yellow solid (yield $42 \%$ ) of $80 \%$ purity. The product was characterized by mass spectrometry and NMR. Exact mass calculated for $\mathrm{C}_{28} \mathrm{H}_{26} \mathrm{O}_{6}$ : 458.2, observed $(\mathrm{M}+\mathrm{H})$ : 459.2 (100\%). ${ }^{1} \mathrm{H}-\mathrm{NMR}$ (500 MHz; DMSO), $\delta 3.82$ (s, 6H), $\delta 3.87$ $(\mathrm{s}, 6 H), \delta 7.03(\mathrm{~d}, 2 H), \delta 7.16(\mathrm{~d}, 2 H), \delta 7.44(\mathrm{~d}, 2 H), \delta 7.56(\mathrm{~d}, 2 H)$, $\delta 7.76(\mathrm{~s}, 1 \mathrm{H}), \delta 7.79(\mathrm{~d}, 1 \mathrm{H}), \delta 7.91(\mathrm{~d}, 1 \mathrm{H}), \delta 8.41(\mathrm{~d}, 2 \mathrm{H})$.

Compound 17 (1,3-bis(3,4-dimethoxystyryl)benzene) was prepared according to Lee et al. (32). Briefly, 1,3-bis(diethoxyphosphinylmethyll benzene was synthesized according to Plater and Jackson (42). $0.7 \mathrm{mmol}$ of 1,3-bis(diethoxyphosphinylmethyl) benzene was resuspended in $50 \mathrm{~mL}$ of anhydrous tetraydrofuran. $2.0 \mathrm{mmol}$ of sodium $t$-butoxide was added and the reaction stirred at $0{ }^{\circ} \mathrm{C}$ for $20 \mathrm{~min}$. Veratraldehyde $(3.6 \mathrm{mmol})$ was then added and the mixture was allowed to stir for another $45 \mathrm{~min}$ at room temperature. About $50 \mathrm{~mL}$ of chilled water was then slowly added over $10 \mathrm{~min}$, and the reaction stirred for a final $30 \mathrm{~min}$ at room temperature. The final mixture was extracted twice with ethyl acetate, and washed once each with $5 \% \mathrm{NaHCO}_{3}$, then brine. A crude column purification was performed in 50:50 ethyl acetate:hexanes to yield three 
separate fractions. A white solid precipitated from the first fraction and was filtered and washed with ethyl acetate (yield 25\%). The final white powder was characterized by mass spectrometry and NMR. Exact mass calculated for $\mathrm{C}_{26} \mathrm{H}_{26} \mathrm{O}_{4}$ : 402.2, observed $\left(\mathrm{M}+\mathrm{Na}^{+}\right): 425.2(100 \%)$. NMR spectra were in agreement with previously published results (32).

\section{Defining linker length and flexibility}

Linker length was quantified by summing the bond lengths according to the following values: $C-C, 1.54 \AA ; C=C, 1.34 \AA ; C-0,1.43 \AA$, and $\mathrm{C}-\mathrm{N}, 1.47 \AA$ (43). Linker flexibility is not based on dynamic simulations but simple quantification of $\mathrm{sp}^{3}$ carbons. Curcumin can be depicted as either a $\beta$-diketone or enol form. The $\beta$-diketone predominates in aqueous environments (44). Based on this finding, we have roughly approximated curcumin as having a single $\mathrm{sp}^{3}$-hybridized carbon center.

\section{Amyloid $\beta$ preparation}

Amyloid $\beta(1-42)$ peptide was purchased from AnaSpec (San Jose, CA, USA). A $1 \mathrm{mg}$ sample of peptide was dissolved in $200 \mu \mathrm{L}$ hexafluoroisopropanol (HFIP) and aliquoted to obtain $0.1 \mathrm{mg}$ stocks. Hexafluoroisopropanol was removed under nitrogen to provide a thin film. Stocks were stored at $-30{ }^{\circ} \mathrm{C}$ until ready-to-use. Immediately prior to the start of the experiment, an aliquot was first fully resuspended in DMSO followed by PBS (pH 7.4) to a final concentration of $25 \mu \mathrm{M}(10 \%$ final DMSO concentration). Aliqouts were then sonicated for $1 \mathrm{~min}$ at room temperature and immediately dispensed into 96-well plates (see below).

\section{Thioflavin T assay}

Inhibition of $A \beta(1-42)$ aggregation was measured using a Thioflavin $T$ (ThT)-binding assay. Stocks of each compound (10 mM) were prepared in DMSO and $1 \mu \mathrm{L}$ of each was added to the wells of black, opaque Corning 96-well plates such that the final solvent concentration was $10 \%$. Compounds were tested over a broad concentration range (1 nM to $100 \mu \mathrm{M}$ ) using 8-11 concentration points. Each concentration was prepared in independent triplicates and a solvent control was included. $9 \mu \mathrm{L}$ of $25 \mu \mathrm{M} \mathrm{A} \beta(1-42)$ sample (see $\mathrm{A} \beta$ preparation above) was added to each well and the samples mixed by gentle tapping. Plates were covered to minimize evaporation and incubated in the dark at room temperature for $46-48 \mathrm{~h}$ with no agitation. After the incubation period, $200 \mu \mathrm{L}$ of $5 \mu \mathrm{M}$ ThT in $50 \mathrm{~mm}$ glycine (pH 8.0) was added to each well. Fluorescence was measured on a SpectraMax M5 (Molecular Devices, Sunnyvale, CA, USA) multi-mode plate reader with excitation and emission wavelengths at $446 \mathrm{~nm}$ and $490 \mathrm{~nm}$, respectively. Data were fit by nonlinear regression analysis using GRAPHPAD PRISM software.

\section{Results and Discussion}

To establish the contribution of each structural module to activity, we assembled a collection of compounds that resemble portions of the curcumin scaffold. Some of these molecules were gathered from the literature and others were built from simple components in a 'bottom-up' synthetic approach (Figure 2). We envisioned that this library could be used to address the three prominent structural features, $R_{1}, R_{2}$, and $R_{3}$, which are observed in naturally occurring ligands. To resolve the contribution of these features, comparisons were made between compounds that, as best as possible, isolate a single chemical signature while retaining the character of the remaining two. To test the activity of compounds in this collection, we employed the well known ThT assay; this dye is fluorescent in the presence of $A \beta$ aggregates and it is commonly used to characterize inhibitors $(45,46)$. In these experiments, we examined the half-maximal concentration $\left(I \mathrm{C}_{50}\right)$ required and expressed these values as relative potency $\left(1 / / \mathrm{C}_{50}\right)$. By comparing the potency of various library

(A)<smiles>COc1cc(C=O)ccc1O</smiles>

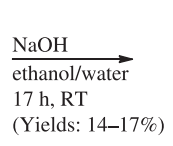<smiles>COc1cc(/C=C/C(=O)c2ccccc2)ccc1O</smiles>

(B)

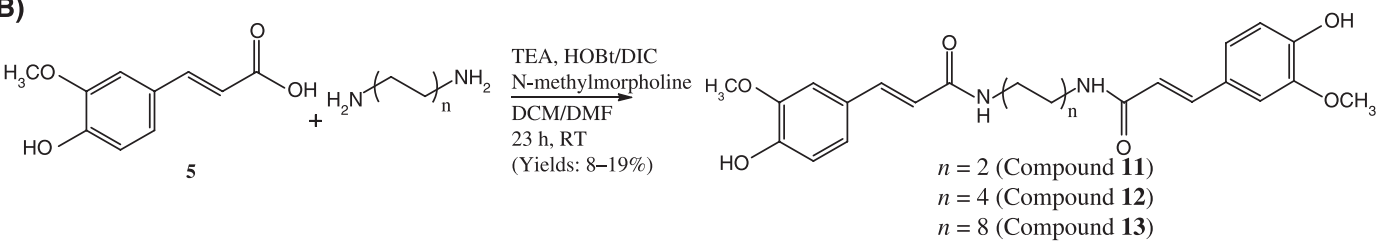

(C)

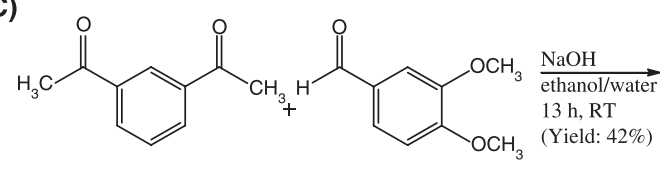<smiles>COc1ccc(/C=C/C(=O)c2cccc(C(=O)/C=C/c3ccc(OC)c(OC)c3)c2)cc1OC</smiles>

Figure 2: Synthesis of curcumin mimetics. (A) Addition of acetophenone to vanillin (1) in basic conditions afforded the aldol condensation product (6). (B) Amide coupling of ferulic acid (5) to alkyl diamines provided the desired compounds (11-13). (C) Similarly, compound (15) was synthesized via aldol condensation by the addition of excess 3,4-dimethoxybenzaldehyde to diacetylbenzene. 


\section{Reinke and Gestwicki}

members, we hoped to provide insight into the relative importance of the different structural features.

\section{$R_{1}$ component: compounds lacking a second terminal aromatic group do not inhibit $A \beta$ aggregation}

Curcumin is composed of two relatively polar aromatic groups connected by a rigid linker. Thus, in our first experiments, we asked whether both aromatic groups were required for activity. Using the ThT assay, we found that simple compounds with a single aromatic group (1-5) did not decrease aggregation of $25 \mu \mathrm{M} A \beta(1-42)$, even at high $(500 \mu \mathrm{M})$ concentrations (Table 1). Conversely, curcumin inhibits $A \beta$ aggregation (IC $\mathrm{C}_{50}$ : approximately $\left.10 \mu \mathrm{M}\right)$, consistent with previous findings $(26,28)$. These data demonstrate that compounds lacking a second phenyl are less potent inhibitors of aggregation.

\section{$R_{2}$ component: substitutions on the aromatic end groups are important for activity}

Curcumin contains a 3-methoxy-4-hydroxy substitution pattern on its aromatic end groups. Similarly, CR displays sulfonic acids in an equivalent position and CG has pendant carboxylates. Thus, we were interested in learning if polar, hydrogen-bonding groups are required. To test this hypothesis, the activities of compounds 15-17 were assayed. Compound $\mathbf{1 6}$ has the same substitution pattern as curcumin, and, similarly, inhibits aggregation with an $\mathrm{IC}_{50}$ value of $0.5 \mu \mathrm{M}$ (32). Compound $\mathbf{1 7}$ is identical to $\mathbf{1 6}$ except that it displays a 3,4-dimethoxy pattern and, interestingly, it has no activity when tested at concentrations up to $100 \mu \mathrm{m}$ (Figure 3). To further explore this requirement, we assembled compound $\mathbf{1 5}$ by a dual aldol condensation (Figure 2). This molecule has a chemical scaffold that resembles curcumin, except that it lacks the hydroxyl substitutions and, like compound 17, it is also inactive (Figure 3). Thus, these results suggest that aromatic substitutions capable of taking part in hydrogen bonding are important for activity. This finding might have been predicted by the invariance of these groups in the natural amyloid ligands; however, these studies demonstrate that these groups serve a functional role and are neither coincidental nor present solely for improved aqueous solubility.

Several other known inhibitors support these observations; compound 9, with two hydroxyl substitutions, blocks $A \beta$ aggregation $\left(\mathrm{IC}_{50}=10 \mu \mathrm{M} ; 36,37\right)$ and compound $\mathbf{1 0}$ has also been shown to significantly reduce fibril formation (35). Together, the results of the $R_{2}$ study suggest that the aromatic end groups require one or more polar, hydrogen bonding substitutions for optimal inhibition of $A \beta$ aggregation.

\section{$R_{3}$ component: length and flexibility of the linker region define inhibitory capacity}

\section{Linker length}

The distance between the terminal aromatic regions of curcumin, $\mathrm{CR}$, and CG are all strikingly similar. Based on this observation, we hypothesized that the length of the linker would be important for activity. To test this idea, we compared the potency of a series of ligands that vary in linker length. To systematically assess the effect of this feature, we constructed a series of ferulic acid dimers. Ferulic acid (5) was used because it contains the same 3,4-substitution pattern as curcumin and, to sample a range of lengths, compounds 11-14 were synthesized via amide coupling of $\mathbf{5}$ to alkyl diamines containing two, four, or eight
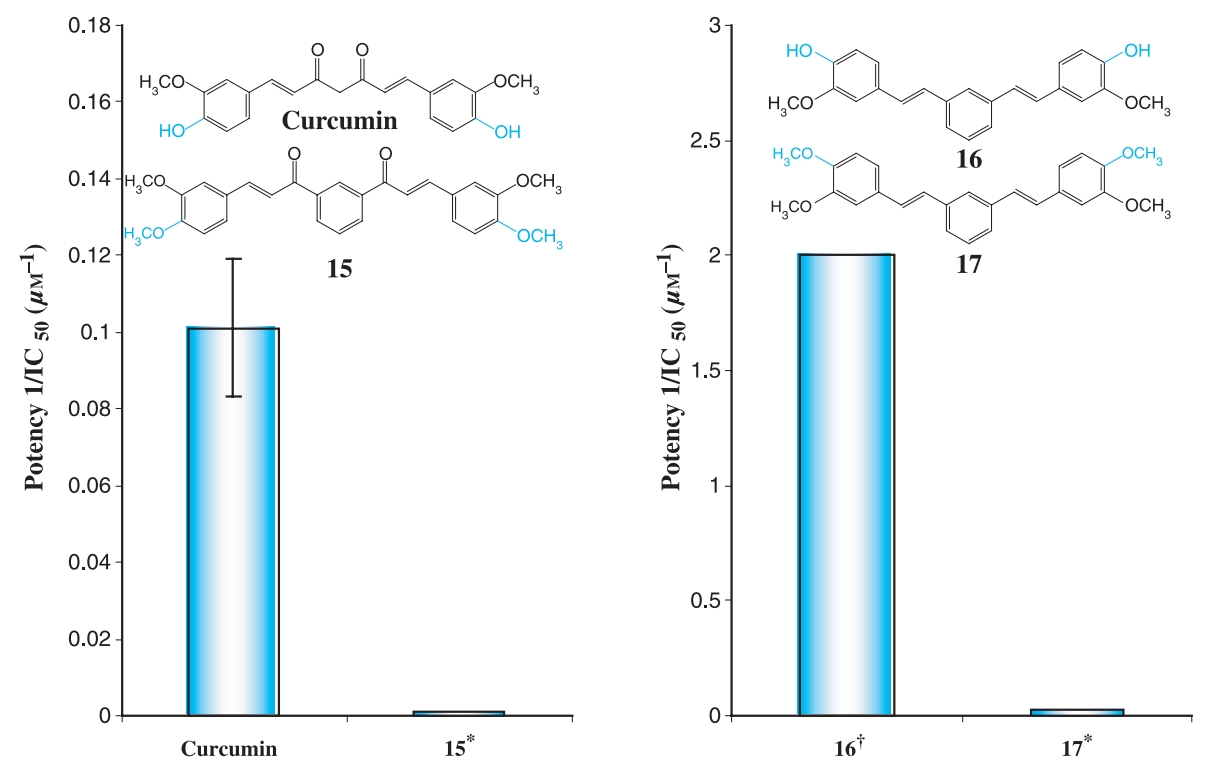

Figure 3: Hydroxy substitutions on the aromatic end groups are required for inhibition. Dimethoxy-substituted compounds (15 and 17) did not inhibit aggregation as well as those with hydroxy substitutions. Potency values $\left(1 / \mathrm{IC}_{50}\right)$ were calculated from eight concentrations of compound (up to $100 \mathrm{~mm}$ ) against $25 \mathrm{~A} \beta(1-42)$. Each concentration was plated in triplicate and error is expressed as standard deviation. Asterisk $\left.{ }^{*}\right)$ indicates $\mathrm{IC}_{50}$ is $>100 \mu \mathrm{M}$. $\dagger \mid \mathrm{C}_{50}$ value reported by Lee et al. (28). 
carbons (Figure 2). Purified yields ranged from $8 \%$ to $19 \%$ and these coupling efficiencies were primarily limited by the poor solubility of ferulic acid and the resulting dimers. Despite these modest yields, this synthetic method provided sufficient material to explore the effects of linker length on potency.

Interestingly, of the four compounds in this series, only the ethyldiamide dimer (11), with a linker length of approximately $16 \AA$, had appreciable activity $\left(\mathrm{IC}_{50}=91 \mu \mathrm{M}\right)$. Compounds 12-14, with linkers ranging from 19 to $26 \AA$ displayed no activity when tested up to $100 \mu \mathrm{M}$ (Figure 4). These results suggest that the activity of curcumin-like compounds is restricted to those with a length of roughly 16-19 A or shorter. To examine the lower limit, we synthesized compounds $\mathbf{6}$ and $\mathbf{7}$ using an aldol condensation (Figure 2). Both these compounds have linkers of approximately $6 \AA$ and they each failed to inhibit fibril formation when tested up to $100 \mu \mathrm{m}$. Interestingly, both compounds meet the requirements proposed by the $R_{1}$ and $R_{2}$ studies, but their activity appears to be limited by
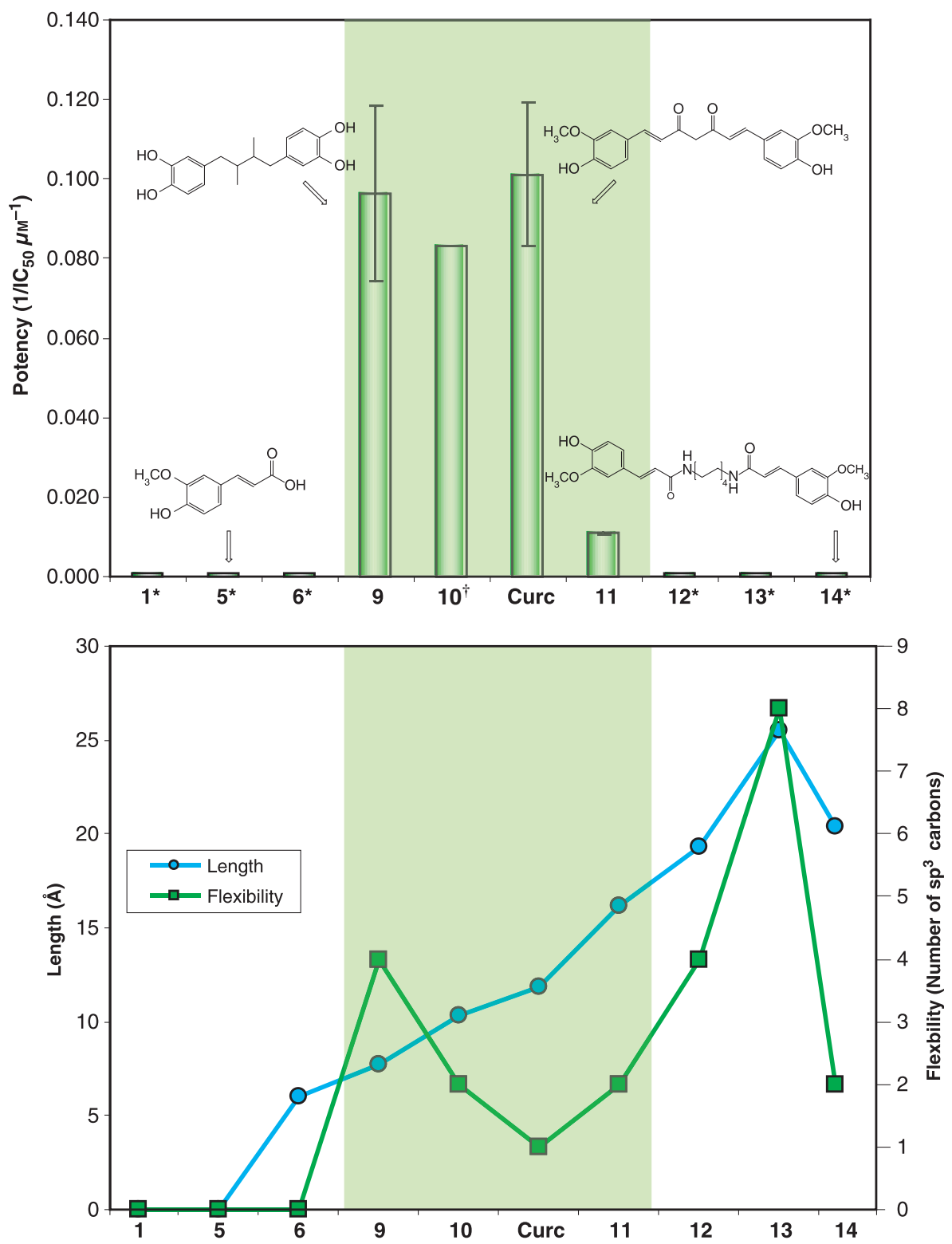

Figure 4: Linker length and flexibility define a narrow region of optimal potency. (A) Linker length and flexibility strongly influence potency. A narrow range of linker length and flexibility defines the optimal window for amyloid beta (A $\beta$ ) inhibition (shaded region). Representative chemical structures are shown, and all compounds were tested up to $100 \mathrm{~mm}$ against $25 \mu \mathrm{M} \mathrm{A} \beta(1-42)$ in triplicate. Error is expressed as standard deviation. Asterisk $\left({ }^{*}\right)$ indicates $I_{50}$ is $>100 \mathrm{mM}$. $\dagger \mathrm{I}_{50}$ value reported by Masuda et al. (31). (B) Quantitative comparison of linker length and flexibility. Linker length (blue) and flexibility (green) are compared. Compounds with optimal activity are in the shaded region. Activity decreases when linker is too short, too long, or too flexible. See Methods and Materials for quantitation methods. 


\section{Reinke and Gestwicki}

their short lengths. Inhibition returned in compounds with lengths of approximately $8-10 \AA$, as shown by compounds 9 and 10 (35). Based on these results, we propose a lower constraint of approximately 6-8 $\AA$. Thus, by creating and testing curcumin-like compounds of varying lengths, we identified upper and lower bounds (Figure 4, shaded region).

\section{Linker flexibility}

The notably rigid quality of natural $A \beta$ ligands led us to predict that flexibility might also influence activity. To facilitate this analysis, relative flexibility was approximated by comparing the total number of freely rotating $\mathrm{sp}^{3}$-hybridized carbons within the backbone. For example, compound $\mathbf{1 1}$ contains two such carbons and is therefore less rigid than curcumin. As mentioned above, this compound inhibits $\mathrm{A} \beta$ aggregation $\left(I C_{50}=91 \mu \mathrm{M}\right)$, although the potency is weak. Increasingly flexible compounds, such as the butyl-diamide dimer (12) with four $\mathrm{sp}^{3}$-hybridized carbons, had no activity. Conversely, rigid molecules, including $\mathbf{9}$ and $\mathbf{1 0}$, are good inhibitors (35). These findings suggest that the linker has a low tolerance for more than one or two $\mathrm{sp}^{3}$-hybridized carbons.

During the course of these experiments, we noticed that length and flexibility are not independent variables. For example, the metaxylene-based dimer (14) has a rigid backbone with only two $s p^{3}$ hybridized carbons, yet, its long linker length appears to prevent this molecule from inhibiting aggregation. Moreover, compounds 6 and $\mathbf{7}$ are rigid, but the backbone of these molecules is only about $6 \AA$; thus, they do not meet the length requirement. Together, we conclude that both the length and flexibility of the linker region strictly define the activity of curcumin-like amyloid inhibitors (Figure 4).

\section{Conclusions and Future Directions}

Striking structural similarities are readily observed in naturally occurring amyloid ligands, such as curcumin, CR, and CG; however,

\section{(A)}

\section{Features important for activity of curcumin-inspired $\mathrm{A} \beta$ ligands}

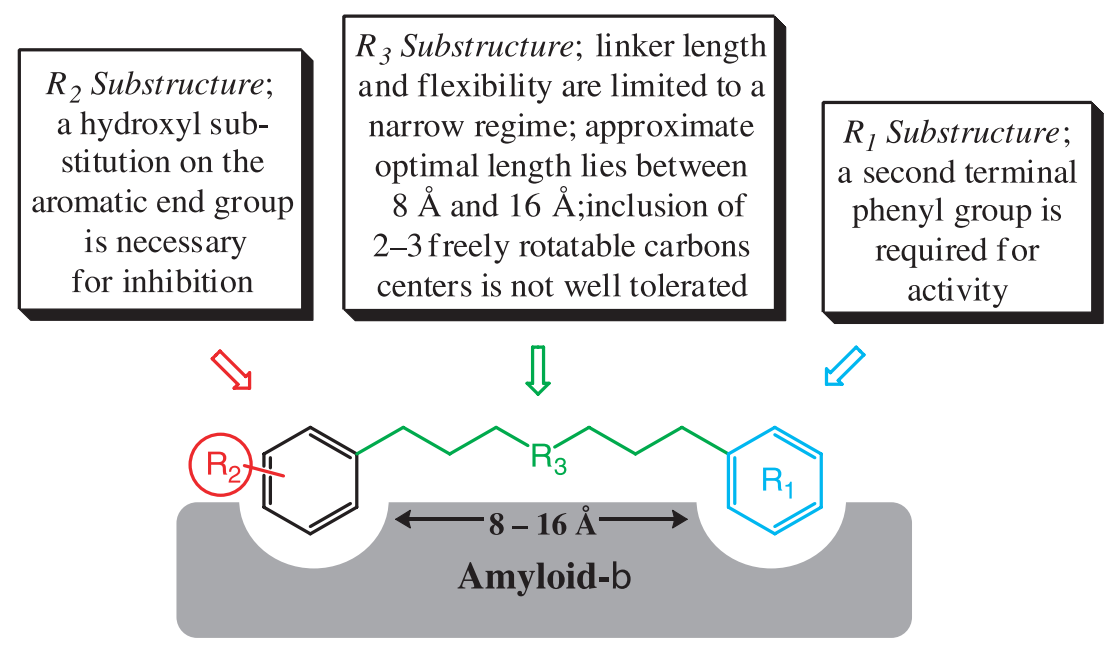

(B) "Goldie-Locks" model showing how linker characteristics contribute to potency
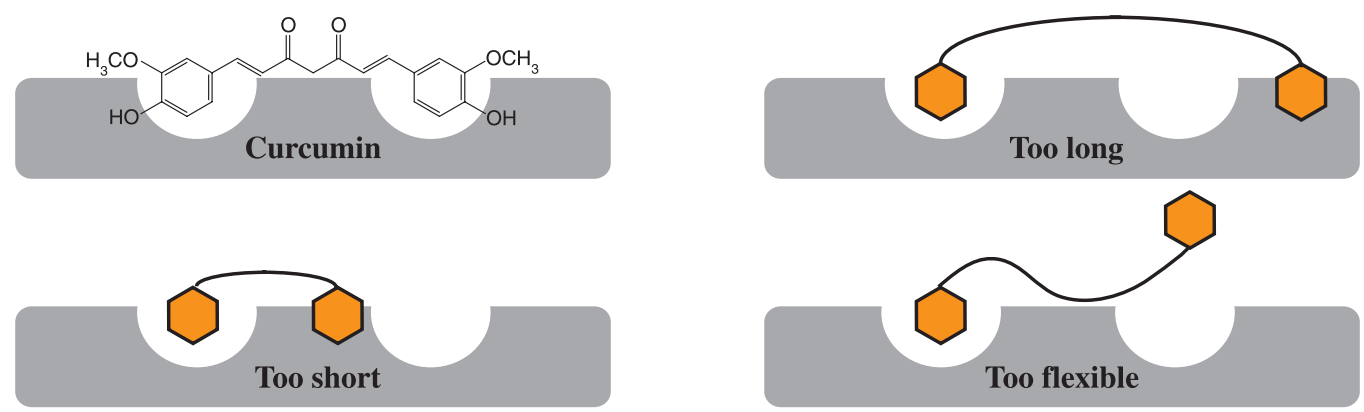

Figure 5: Three structural features of curcumin-like compounds that are important for activity. (A) Schematic depiction of the three regions $\left(R_{1}, R_{2}\right.$, and $\left.R_{3}\right)$ that were identified and the approximate features that describe potent ligands. (B) Goldie-Locks model. Based on our results, we propose that the substitution pattern and linker properties of curcumin are just right. Importantly, the length and flexibility of the linker both contribute to defining the optimal range. 
SAR of Amyloid Beta-aggregation Inhibitors

a systematic analysis of these perceived commonalities had not been performed. Thus, the main goal of this study was to understand which features of these inhibitors are critical for activity. As part of these experiments, we hoped to provide context in which to explain the activity of previously described, synthetic inhibitors (29-38). In addition, we hoped that these studies might guide the rational design of new compounds.

Our approach was to assay a library of compounds that collectively address three distinct structural features that were predicted to be critical for inhibition of $A \beta$ aggregation. Our findings indicate that alteration or elimination of any one of the three major components results in a significant loss of activity (Figure 5A). First, in the $R_{1}$ study, we found that simple aromatics do not have activity. It is worth noting that, although the majority of known amyloid ligands have at least two phenyl groups, a handful of compounds with a single phenyl ring have been shown to inhibit $A \beta$ by other experimental approaches $(29,47)$. These observations are not necessarily in conflict, because it is likely that distinct experimental platforms (e.g. ThT, direct binding, etc.) and subtle changes in handling (e.g. different buffers, temperatures, time, etc.) may give rise to different outcomes. In the second portion of our analyses, we found that loss of the hydroxyl group on the aromatic rings abolishes activity. This result is consistent with the architecture of natural amyloid ligands, such as curcumin and CR. In addition, polar functional groups (and often, hydroxyls) are commonly seen in other reported ligands $(26,29,33,35)$. Our results suggest that these features are indispensable for activity.

Finally, results from the $R_{3}$ study establish that the optimal linker region is restricted to a defined length and rigidity. Specifically, we found that ligands containing linkers between 8 and $16 \AA$ that are rigid (less than one to two freely rotating carbons) are the best inhibitors. Interestingly, many of the best amyloid ligands fall within the observed optimal range. For example, CR, CG, RS-0406, and rosmarinic acid all meet the requirements $(23-25,35,38)$. In addition, the linker component seems to be one of the most significant features, because compounds that fulfill the other substructural requirements, such as aromatic substitution, still fail to inhibit if they have a short linker. An interesting exception to this observation is compound $\mathbf{8}$ (resveratrol; $\left.\mathrm{IC}_{50}=11 \mu \mathrm{M}\right)$. Despite its short linker length, this compound is active, suggesting a possible alternative mechanism for inhibition or binding to a different site on the amyloid, such as the site occupied by ThT. Despite this finding, our studies show that the length and flexibility of the linker is, in general, a critical component of potent inhibitors.

Global analysis of the three features allows us to construct a preliminary model for how ligands related to curcumin might interact with the $A \beta$ surface. From the $R_{1}$ study, we predict that there should be at least two binding sites to accommodate the aromatic end groups (Figure $5 \mathrm{~B}$ ). The $\mathrm{A} \beta$ peptide contains a number of aromatic (e.g. Phe4, Phe19, and Phe20) and other hydrophobic residues that are critical for aggregation $(48,49)$; thus, it is reasonable to predict that there are adequate opportunities for functionally important hydrophobic interactions. In turn, the $R_{2}$ study predicts that there might be good options for hydrogen bonding within these pockets, but the nature of these contacts is not clear. One of the interesting aspects of this study is that it provides an approximate distance between the docking sites. Specifically, we found that lengths below $6-8 \AA$ and beyond $16-19 \AA$ are not well tolerated, so the sites likely lie between 8 and $16 \AA$ from each other. There are probably at least two pockets involved in defining this distance, but it is unclear if multiple primary and/or secondary interactions are involved. Regardless, to emphasize the strict requirements for optimal binding and the diminished activity outside this zone, we refer to this illustration as the 'Goldie-Locks' model (Figure 5B). In the model, too much flexibility (e.g. $>2$ freely rotating centers) allows for greater sampling of chemical space by the second aromatic group and, as a result, the high entropic penalty paid for docking this group disfavors binding. Importantly, none of these requirements describe the features of thiazolidine- or benzothiazoleclass inhibitors (50-52), which are typically short, uncharged, and relatively non-polar, and it seems likely that a different model applies to these compounds. This conclusion is consistent with recent evidence that there are at least two distinct sites for small molecules on amyloids $(39,53-55)$. Moreover, as implied above, it is possible that resveratrol, which is an outlier in our study, may access this alternative site. Together, these studies provide a model for how organic compounds bind the surface of amyloids and prevent self-assembly.

We have used a collection of curcumin-inspired ligands to chemically 'map' the $A \beta$ surface. We predict that further studies into the interaction between small molecules and $A \beta$ will provide information that will supplement the work emerging from structural studies. These findings may contribute to our understanding of the features that propagate aggregation and, perhaps, encode for neurotoxicity and, together, these studies might inform those seeking better inhibitors and chemical probes.

\section{Acknowledgments}

The authors thank C. G. Evans and E. S. Underbakke for critical reading of the manuscript and $\mathrm{C}$. Gaines for stimulating discussions. This work was supported by a Pilot Grant from the University of Michigan's Alzheimer's Disease Research Center.

\section{References}

1. Selkoe D.J. (1991) The molecular pathology of Alzheimer's disease. Neuron;6:487-498.

2. Caughey B., Lansbury P.T. (2003) Protofibrils, pores, fibrils, and neurodegeneration: separating the responsible protein aggregates from the innocent bystanders. Ann Rev Neurosci;26:267298.

3. Glabe C.C. (2005) Amyloid accumulation and pathogensis of Alzheimer's disease: significance of monomeric, oligomeric and fibrillar Abeta. Biochemistry;38:167-177.

4. Jarrett J.T., Lansbury P.T. Jr (1993) Seeding "one-dimensional crystallization" of amyloid: a pathogenic mechanism in Alzheimer's disease and scrapie? Cell;73:1055-1058. 


\section{Reinke and Gestwicki}

5. Morgan C., Colombres M., Nunez M.T., Inestrosa N.C. (2004) Structure and function of amyloid in Alzheimer's disease. Progress Neurobiol;74:323-349.

6. Smith T.J., Stains C.I., Meyer S.C., Ghosh I. (2005) Inhibition of beta-amyloid fibrillization by directed evolution of a beta-sheet presenting miniature protein. J Am Chem Soc;128:14456-14457.

7. Urbanc B., Cruz L., Teplow D.B., Stanley H.E. (2006) Computer simulations of Alzheimer's amyloid beta-protein folding and assembly. Current Alzheimers Res;3:493-504.

8. Haass C., Schlossmacher M.G., Hung A.Y., Vigo-Pelfrey C., MelIon A., Ostaszewski B.L. et al. (1992) Amyloid beta-peptide is produced by cultured cells during normal metabolism. Nature;359:322-325.

9. Ghosh A.K., Kumaragurubaran N., Tang J. (2005) Recent developments of structure based beta-secretase inhibitors for Alzheimer's disease. Curr Topics Med Chem;5:1609-1622.

10. Glabe C.G., Kayed R. (2006) Common structure and toxic function of amyloid oligomers implies a common mechanism of pathogenesis. Neurology;66:S74-S78.

11. Walsh D.M., Klyubin I., Fadeeva J.V., Rowan M.J., Selkoe D.J. (2002) Amyloid-beta oligomers: their production, toxicity and therapeutic inhibition. Biochem Soc Trans;30:552-557.

12. Barghorn S., Nimmrich V., Striebinger A., Krantz C., Keller P., Janson B. et al. (2005) Globular amyloid beta-peptide oligomer a homogenous and stable neuropathological protein in Alzheimer's disease. J Neurochem;95:834-847.

13. Findeis M.A. (2000) Approaches to discovery and characterization of inhibitors of amyloid beta-peptide polymerization. Biochem Biophys Acta;1502:76-84.

14. Lee V.M. (2002) Amyloid binding ligands as Alzheimer's disease therapies. Neurobiol Aging;23:1039-1042.

15. Cohen F.E., Kelly J.W. (2003) Therapeutic approaches to proteinmisfolding diseases. Nature;426:905-909.

16. Evans C.G., Wisen S., Gestwicki J.E. (2006) Heat shock proteins 70 and 90 inhibit early stages of amyloid beta-(1-42) aggregation in vitro. J Biol Chem;281:33182-33191.

17. Makin O.S., Serpell L.C. (2005) Structures for amyloid fibrils. FEBS J:272:5950-5961.

18. Luhrs T., Ritter C., Adrian M., Riek-Loher D., Bohrmann B.,

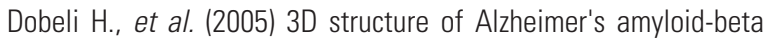
(1-42) fibrils. Proc Natl Acad Sci USA;102:17342-17347.

19. Makabe K., McElheny D., Tereshko V., Hilyard A., Gawlak G., Yan S., et al. (2006) Atomic structures of peptide self-assembly mimics. Proc Natl Acad Sci USA;103:17753-17758.

20. Buchete N.V., Hummer G. (2007) Structure and dynamics of parallel beta-sheets, hydrophobic core, and loops in Alzheimer's A beta fibrils. Biophys J;92:3032-3039.

21. Eisenberg D., Nelson R., Sawaya M.R., Balbirnie M., Sambashivan S., Ivanova M.I. et al. (2006) The structural biology of protein aggregation diseases: Fundamental questions and some answers. Acc Chem Res;39:568-575.

22. Oyler N.A., Tycko R. (2004) Absolute structural constraints on amyloid fibrils from solid-state NMR spectroscopy of partially oriented samples. J Am Chem Soc;126:4478-4479.

23. Klunk W.E., Pettegrew J.W., Abraham D.J. (1989) Quantitative evaluation of congo red binding to amyloid-like proteins with a beta-pleated sheet conformation. J Histochem Cytochem; 37:1273-1281.
24. Lorenzo A., Yankner B.A. (1994) Beta-amyloid neurotoxicity requires fibril formation and is inhibited by congo red. Proc Natl Acad Sci USA;91:12243-12247.

25. Klunk W.E., Debnath M.L., Pettegrew J.W. (1995) Chrysamine-G binding to Alzheimer and control brain: autopsy study of a new amyloid probe. Neurobiology of aging;16:541-548.

26. Yang F., Lim G.P., Begum A.N., Ubeda O.J., Simmons M.R., Ambegaokar S.S. et al. (2005) Curcumin inhibits formation of amyloid beta oligomers and fibrils, binds plaques, and reduces amyloid in vivo. J Biol Chem;280:5892-5901.

27. Ryu E.K., Choe Y.S., Lee K.H., Choi Y., Kim B.T. (2006) Curcumin and dehydrozingerone derivatives: synthesis, radiolabeling, and evaluation for beta-amyloid plaque imaging. $J$ Med Chem;49:6111-6119.

28. Ono K., Hasegawa K., Naiki H., Yamada M. (2004) Curcumin has potent anti-amyloidogenic effects for Alzheimer's beta-amyloid fibrils in vitro. J Neurosci Res;75:742-750.

29. Necula M., Kayed R., Milton S., Glabe C.G. (2007) Small molecule inhibitors of aggregation indicate that amyloid beta oligomerization and fibrillization pathways are independent and distinct. J Biol Chem; 2007 Apr;282:10311-10324.

30. Kung H.F., Lee C.W., Zhuang Z.P., Kung M.P., Hou C., Plossl K. (2001) Novel stilbenes as probes for amyloid plaques. J Am Chem Soc;123:12740-12471.

31. Riviere C., Richard T., Quentin L., Krisa S., Merillon J.M., Monti J.P. (2007) Inhibitory activity of stilbenes on Alzheimer's beta-amyloid fibrils in vitro. Bioorganic \& medicinal chemistry;15:1160-1167.

32. Lee K.H., Shin B.H., Shin K.J., Kim D.J., Yu J. (2005) A hybrid molecule that prohibits amyloid fibrils and alleviates neuronal toxicity induced by beta-amyloid (1-42). Biochem Biophys Res Commun;328:816-823.

33. Porat Y., Abramowitz A., Gazit E. (2006) Inhibition of amyloid fibril formation by polyphenols: structural similarity and aromatic interactions as a common inhibition mechanism. Chem Biol Drug Design;67:27-37.

34. Taniguchi S., Suzuki N., Masuda M., Hisanaga S., Iwatsubo T., Goedert M. et al. (2005) Inhibition of heparin-induced tau filament formation by phenothiazines, polyphenols, and porphyrins. J Biol Chem;280:7614-7623.

35. Masuda M., Suzuki N., Taniguchi S., Oikawa T., Nonaka T., Iwatsubo T. et al. (2006) Small molecule inhibitors of alpha-synuclein filament assembly. Biochemistry;45:6085-6094.

36. Naiki H., Hasegawa K., Yamaguchi I., Nakamura H., Gejyo F., Nakakuki K. (1998) Apolipoprotein E and antioxidants have different mechanisms of inhibiting Alzheimer's beta-amyloid fibril formation in vitro. Biochemistry;37:1782-1789.

37. Moss M.A., Varvel N.H., Nichols M.R., Reed D.K., Rosenberry T.L. (2004) Nordihydroguaiaretic acid does not disaggregate beta-amyloid (1-40) protofibrils but does inhibit growth arising from direct protofibril association. Mol Pharm;66:592-600.

38. Nakagami Y., Nishimura S., Murasugi T., Kaneko I., Meguro M., Marumoto S. et al. (2002) A novel beta-sheet breaker, RS-0406, reverses amyloid beta-induced cytotoxicity and impairment of long-term potentiation in vitro. British J Pharmacol;137:676-682.

39. Ye L., Morgenstern J.L., Gee A.D., Hong G., Brown J., Lockhart A. (2005) Delineation of positron emission tomography imaging agent binding sites on beta-amyloid peptide fibrils. J Biol Chem;280:23599-23604. 
SAR of Amyloid Beta-aggregation Inhibitors

40. Ansari F.L., Nazir S., Noureen H., Mirza B. (2005) Combinatorial synthesis and antibacterial evaluation of an indexed chalcone library. Chem Biodiversity;2:1656-1664.

41. Spasova M., Kortenska-Kancheva V., Totseva I., Ivanova G., Georgiev L., Milkova T. (2006) Synthesis of cinnamoyl and hydroxycinnamoyl amino acid conjugates and evaluation of their antioxidant activity. J Pept Sci;12:369-375.

42. Plater M.J., Jackson T. (2003) Polyaromatic amines. Part 3: Synthesis of poly(diarylamino)styrenes and related compounds. Tetrahedron;59:4673-4685.

43. Hodgman C.D., Veazey W.R., Weast R.C. Chemical Rubber Company. (1998) Handbook of chemistry and physics; a ready-reference book of chemical and physical data. Cleveland: Cleveland Rubber Co.

44. Ortica F., Rodgers M.A. (2001) A laser flash photolysis study of curcumin in dioxane-water mixtures. Photochem Photobiol; 74:745-751.

45. LeVine H. III (1993) Thioflavin T interaction with synthetic Alzheimer's disease beta-amyloid peptides: detection of amyloid aggregation in solution. Prot Sci;2:404-410.

46. LeVine H. III (1999) Quantification of beta-sheet amyloid fibril structures with thioflavin T. Methods Enzymol;309:274-284.

47. De Felice F.G., Vieira M.N., Saraiva L.M., Figueroa-Villar J.D., Garcia-Abreu J., Liu R. et al. (2004) Targeting the neurotoxic species in Alzheimer's disease: inhibitors of Abeta oligomerization. Faseb J;18:1366-1372.

48. Shivaprasad S., Wetzel R. (2006) Scanning cysteine mutagenesis analysis of Abeta-(1-40) amyloid fibrils. J Biol Chem;281:9931000.

49. Williams A.D., Shivaprasad S., Wetzel R. (2006) Alanine scanning mutagenesis of Abeta-(1-40) amyloid fibril stability. J Mol Biol;357:1283-1294.

50. Campiglia P., Esposito C., Scrima M., Gomez-Monterrey I., Bertamino A., Grieco P. et al. (2007) Conformational stability of Abeta-(25-35) in the presence of thiazolidine derivatives. Chem Biol Drug Design;69:111-118.
51. Zhuang Z.P., Kung M.P., Wilson A., Lee C.W., Plossl K., Hou C. et al. (2003) Structure-activity relationship of imidazo[1,2-a]pyridines as ligands for detecting beta-amyloid plaques in the brain. J Med Chem;46:237-243.

52. Chandra R., Kung M.P., Kung H.F. (2006) Design, synthesis, and structure-activity relationship of novel thiophene derivatives for beta-amyloid plaque imaging. Bioorg Med Chem Lett;16:13501352.

53. Lockhart A., Ye L., Judd D.B., Merritt A.T., Lowe P.N., Morgenstern J.L. et al. (2005) Evidence for the presence of three distinct binding sites for the thioflavin $T$ class of Alzheimer's disease PET imaging agents on beta-amyloid peptide fibrils. J Biol Chem;280:7677-7684.

54. LeVine H. 3rd (2005) Mechanism of Abeta(1-40) fibril-induced fluorescence of (trans,trans)-1-bromo-2,5-bis(4-hydroxystyryl)benzene (K114). Biochemistry;44:15937-15943.

55. Nesterov E.E., Skoch J., Hyman B.T., Klunk W.E., Bacskai B.J., Swager T.M. (2005) In vivo optical imaging of amyloid aggregates in brain: design of fluorescent markers. Angew Chem;44:5452-5456.

\section{Supplementary Material}

The following supplementary material is available for this article:

Figure S1. The NMR spectra for compounds 11-14.

This material is available as part of the online article from: http://www.blackwell-synergy.com/doi/abs/10.1111/j.1747-0285.2007. 00557.x (This link will take you to the article abstract).

Please note: Blackwell Publishing are not responsible for the content or functionality of any supplementary materials supplied by the authors. Any queries (other than missing material) should be directed to the corresponding author for the article. 